\title{
Zur Kenntniss des Oroselons und Peucedanins
}

\author{
$\operatorname{von}$ \\ Maximilian Popper.
}

Aus dem I. chemischen Laboratorium der k. k. Universität in Wien.

(Vorgelegt in der Sitzung am 10. Juni 1898.)

In der Absicht, eine nähere Untersuchung des Oroselons auszuführen, habe ich käufliches Peucedanin von der Firma E. Merck in Darmstadt der Einwirkung von Salzsäure unterworfen, um Oroselon darzustellen. Das erste von Merck bezogene Präparat lieferte reichliche Mengen dieses Körpers; jedoch eine zweite Sendung von Peucedanin, welche wohl einen dem verlangten naheliegenden Schmelzpunkt besass und bei der Analyse Zahlen lieferte (C...70.2\%, H... 5.5\%), welche mit den für Peucedanin gerechneten in naher Übereinstimmung stehen, gab bei Behandlung mit Salzsäure aber keine Ausscheidung von Oroselon, sondern lieferte ein sandiges Zersetzungsproduct, welches schon im Äusseren keine Ähnlichkeit mit Oroselon zeigte und sich von diesem durch den Schmelzpunkt unterschied. Beim Erhitzen auf circa $200^{\circ}$ färbte es sich dunkel, bei $240^{\circ}$ verflüssigte es sicn unter totaler Zersetzung; Oroselon hingegen schmilzt bei $177^{\circ}$.

Auch die Verbrennung dieses Productes lieferte Werthe, die von den erforderten völlig verschieden sind (C . . 64 $84 \%$, H. . 2. $92 \%$; Oroselon verlangt: C . . 68.85\%, H. .4.91\%). Auf eine Anfrage bei der Firma Merck, ob das gelieferte Peucedanin wirklich aus Peucedanum officinale hergestellt sei. wurde mir die Antwort zu Theil, dass dasselbe aus der Wurzel von Imperatoria Ostruthium dargestellt worden war. 
Das von Schlatter entdeckte, von Bothe. Erdmann" und Anderen später untersuchte Peucedanin wurde stets aus Pencedanum officinale dargestellt. Erst Wagner hat das ron Osann und Wackenroder ${ }^{5}$ in der Wurzel von Imperatoria Ostruthitum aufgefundene Imperatorin mit dem Peucedanin identificirt. Diese Identitätserklärung stützt sich auf die Ühereinstimmung der physikalischen Eigenschaften, auf die gleiche chemische Zusammensetzung und auf den Umstand, dass beide Producte bei der Behandlung mit Ätzkali neben Angelicasäure Oroselon Iiefern. Die Identität des Peucedanins mit Imperatorin warde von Gorup-Besanez gelegentlich seiner Untersuchung über das Ostruthin, ${ }^{6}$ welches ebenfalls aus Imperatoria Ostruthitm erhalten wurde, jedoch in Zweifel gezogen, und er bemerkt am Schlusse seiner Abhandlung: "Jedenfalls wird man zuge. stehen müssen, dass die Fundamente, auf welchen die Lehre von der Identität des Peucedanins und Imperatorins ruht, ziemlich unsichere sind $*$.

Kurze Zeit darauf haben endlich Hlasiwetz und Weide ${ }^{7}$ gezeigt, dass das Peucedanin bei Behandlung mit Ätzkali keine Angelicasäure abzuspalten vermag, wodurch selbstverständlich eine der gewichtigsten Stützen für eine Identität desselben mit Imperatorin hinfällig wurde.

Bei dieser Sachlage schien es mir von Wichtigkeit, die Darstellung des Peucedanins selbst vorzunehmen. $\mathrm{Zu}$ diesem Ende habe ich mir eine grössere Quantität der Wurzel von Pencedanum officinale durch eine renommirte Droguenhandlung verschafft. Bevor ich an die Aufarbeitung derselben herantrat, wurden Proben der Wurzel von Herrn Dr. Hockauf im pharmacologischen Institute des Herrn Hofrathes Vogl vorgenommen, durch welche der Beweis erbracht wurde, dass die in Arbeit genommene Wurzel auch von Pencedanum officinale

1 Annalen der Pharm., 5, 201.

2 Journal für prakt. Chemie, $46,371$.

3 Journal für prakit. Chemie, 16, 42.

+ Journal für prakt. Chemie, 61, 503; 62, 275.

5 Arch. der Pharm. 37, 341.

(5) Berl. Ber., VII, 568.

$\checkmark$ Annalen der Chemie, 174,67. 
stammte. Herr Dr. Hockauf theilt über seine Untersuchung Folgendes mit:

"Die mir übergebene, walzlich-spindelige, mehrköpfige, von Blattscheidenresten schopfige, dicke Wurzel von Peucedanum officinale Linn. kommt der Länge nach gespalten, in Stücken von 6--8 cm Länge, 2-31/2 $\mathrm{cm}$ im Durchmesser, in den Handel. Sie ist sowohl an den oft verlängerten Köpfen, als auch am Körper dicht quergeringelt oder querrunzelig, dunkelbraun bis schwärzlich, unter dem abgeschabten Periderma gelblichweiss. Der Geruch ist eigenthümlich widrig, fast ranzig, der Geschmack scharf und bitter.

Am Wurzelkopfe beträgt der Querschnitt der Rinde nahezu ein Drittel des Halbmessers; dieselbe ist grob radial gestreift, die Streifen sind gelbbräunlich, von zahlreichen braungelben Secretgängen dicht punctirt. In der Peripherie ist sie locker und lückig. Der Holzkörper, von nahezu gleicher Breite wie die Rinde, ist weiss, in der Peripherie gelbbraun, dicht radial gestreift und zerklüftet; die Holzstrahlen sind gelb mit undeutlich wahrnehmbaren Gefässpunkten, während das Mark weiss, locker, von zerstreuten, gelbbraunen Balsamcanälen punctirt erscheint.

Unter dem Mikroskope besteht die Aussenrinde aus einem mehrreihigen Periderm, die äusseren Partien mit einem rothbraunen Inhalte, die inneren farblos; darauf folgt in der mehr schmaklen Mittelrinde ein lückiges Parenchym von anfangs mehr collabirten, tangential gestreckten Zellen, darauf grössere, rundliche, welche die bereits äusserlich wahrnehmbaren Secretbehälter in 2-3 Reihen umgeben. Die Balsamcanäle sind im Querschnitte rundlich, elliptisch, oft tangential gestreckt, $150 \mu$ bis $300 \mu \mathrm{im}$ Durchmesser, ausgekleidet mit einem dünnwandigen, lückenlos schliessenden Epithel; im Längsschnitte langgestreckt, schlauchförmig, manchmal gabelig. Sie führen als Inhalt Massen und Haufwerk von nadel- oder keilförmigen, farblosen Krystallen und gelbbraune Balsamtropfen, welche oft, mit zahlreichen Kryställchen bedeckt, igelartig aussehen (Peucedanin?).

Im Baststrahle nehmen die etwas kleineren Balsamcanäle $(80-160 \mu)$ die Mitte ein, sind meist radial geordnet, umgeben 
ron zwei bis drei Reihen rundlicher Parenchymzellen, flankirt einer- oder beiderseits von Strängen zusammengefallener Siebröhren, welche meist durch Querstränge zwischen den Secretbehältern untereinander in Verbindung stehen. Das Rindenparenchym zeigt zahlreiche, drei- bis viereckige Intercellularen; die Zellen sind rundlich, dünnwandig, 80-100 im Durchmesser; die Wände quellen etwas im Wasser, fürben sich mit Hämatoxylin sofort schön violett; sie sind strotzend gefüllt mit kleinkörniger $(9-12 \mu)$, componirter Stärke.

Die Markstrahlen, welche Innenrinde und Holzkörper durchziehen, sind 3--6 Zellen breit; die Zellen sind im Querschnitte radial gestreckt, dünnwandig. mit kleinkörniger, componirter Stärke straff gefüllt. Ein Reihencambium trennt die Rinde rom Holzkörper. Die Gefässe sind im Längsschnitte wurmförmig gekrümmt und oft netzfürmig getüpfelt. Im Querschnitte stehen sie in ein bis zwei Reihen radial angeordnet und haben $40-60 \mu \mathrm{im}$ Durchmesser.

Das Holzparenchym besteht aus dünnwandigen Zellen, 24-40 $\mu$ im Durchmesser. Im Wurzelkopfe sind auch Libriformbündel in den Gefässtheil eingebettet.

Das Mark besteht aus einem lockeren Parenchym dünnwandiger, rundlichér Zellen, welche mit kleinkörniger, componirter Stärke reichlich gefüllt sind. Gegen den Holzkörper zu liegen ähnlich gebaute und ebenso grosse Secretbebälter wie in der Mittelrinde.

Der Wassergehait des Wurzelpulvers beträgt $8 \cdot 94 \%$, die Gesammtasche $4 \cdot 487^{\circ} \%$, die Reinasche $3 \cdot 387^{\circ}{ }^{\circ}{ }^{\mu}$.

Die Darstellung des Peucedanins aus der Wurzel habe ich

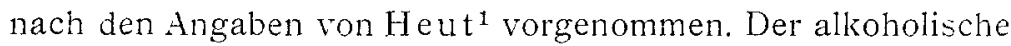
Extract schied nach einigen Tagen eine reichliche Menge von Krystallen ab; diese dicke Nasse wurde mit wenig Alkohol angerührt, auf Filter gebracht und abgesaugt. Zur vorläufigen Reinigung habe ich das Rohproduct aus Alkohol, dann aus Petroleumäther umkrystallisirt.

Man erhält so eine schwach gelblichweisse, aus feinen. seidenglänzenden Nadeln bestehende Krystallmasse, die bei

1 Annalen der Chemie, 176, 71. 
der Temperatur ron circa $80^{\circ}$ zu erweichen beginnt und etwas über $100^{\circ}$ vollständig geschmolzen ist. Aus dieser Thatsache ist der Schluss zu ziehen, dass dieses Product keineswegs einheitlich ist, und ich habe nun zunächst getrachtet, durch Anwendung verschiedener Lösungsmittel eine Trennung der Bestandtheile herbeizuführen.

Mit Hilfe von kaltem, absoluten Äther gelang es, eine schwerlösliche Partie $A$ von einer leichter löslichen $B$ abzutrennen, wenn man die fein zerriebenen Rohkrystalle längere Zeit mit grossen Quantitäten dieses Lösungsmittels schüttelt. Diese beiden Fractionen erwiesen sich auch different im Schmelzpunkt. $A$ schmilzt bei $84-93^{\circ} \mathrm{C}$, während $B$ bei $93^{\circ}$ bis $104^{\circ}$ sich verflüssigt. Durch systematisches fractionirtes Umkrystallisiren der Partie $B$, Vereinigung der Fractionen von gleichem Schmelzpunkt und Wiederkrystallisiren derselben aus verschiedenen Mitteln, wie Äther, Benzol, Petroleumäther, konnte endlich ein bei $104-105^{\circ} \mathrm{C}$. (uncorr.) schmelzendes Product abgeschieden werden, welches in seinem äusseren Ansehen den Beschreibungen, wie sie Heut, Hlasiwetz und Weidel vom Peucedanin geben, zwar entspricht, sich aber in seiner Zusammensetzung von jenem unterscheidet. Diese bei $105^{\circ}$ schmelzende Substanz bildet schwach gelblichweisse, glänzende, dünne Krystallnadeln, die nicht selten zu concentrisch gruppirten Drusen verwachsen sind. Die Verbindung ist bei $17 \mathrm{~mm}$ Druck und der Temperatur von $276-281^{\circ} \mathrm{C}$. (uncorr,) unzersetzt destillirbar; das Destillat erstarrt und krystallisirt aus einer ätherischen Lösung in den früher beschriebenen Formen aus.

Das Product ist, gleich dem Peucedanin, in Kaliauge mit gelber Farbe löslich und kann aus dieser Lösung durch Säuren wieder abgeschieden werden.

Die Verbrennung der im Vacuum zur Gewichtsconstanz getrockneten Substanz ergab folgende Werthe:

$0.2494 \mathrm{~g}$ Substanz gaben $0.6395 \mathrm{~g}$ Kohlensäure und $0.1256 \mathrm{~g}$ Wasser.

In 100 Theilen:

C......69.93

H...... 5.59. 
Vergleicht man diese Zahlen mit jenen, welche von Bothe, ${ }^{1}$ Wagner, ${ }^{2}$ Hlasiwetz und Weidel ${ }^{3}$ für die Zusammensetzung des Peucedanins ermittelt wurden, so zeigen sich erhebliche Differenzen:

\begin{tabular}{|c|c|c|c|c|}
\hline $\mathrm{C}_{106} \mathrm{H}_{10} \mathrm{O}_{4}$ & Bothe & Wagner & $\begin{array}{l}\text { H1asiwetz } \\
\text { und Weidel }\end{array}$ & Popper \\
\hline$\ldots .70 \cdot 5$ & $70 \cdot 6$ & $70 \cdot 6$ & $70 \cdot 4 \quad 70 \cdot 6$ & $69 \cdot 93$ \\
\hline$\ldots .5 \cdot 8$ & $6 \cdot 0$ & $5 \cdot 9$ & $5 \cdot 9$ & $5 \cdot 59$ \\
\hline
\end{tabular}

Da die Verbindung bei Behandlung mit Salzsäure oder Jodwasserstoffsäure Methyl abspaltet und Oroselon bildet, habe ich eine Methoxylbestimmung nach der Zeisel'schen Methode vorgenommen und dabei folgendes Resultat erhalten:

$0 \cdot 2658 g$ Substanz gaben $0.2430 g$ Jodsilber.

In 100 Theilen: $\mathrm{OCH}_{3} \ldots 12 \cdot 12$.

Mit Rücksicht auf dieses Resultat lässt sich aus der früher mitgetheilten Verbrennung für die Substanz die Formel $\mathrm{C}_{15} \mathrm{H}_{14} \mathrm{O}_{4}$ ableiten. Dieselbe vetlangt in 100 Theilen:

\begin{tabular}{|c|c|c|}
\hline & Berechnet & Gefunden \\
\hline C. & $69 \cdot 76$ & $69 \cdot 93$ \\
\hline & $5 \cdot 42$ & $5 \cdot 59$ \\
\hline $\mathrm{OCH}$ & $12 \cdot 01$ & $12 \cdot 12$ \\
\hline
\end{tabular}

Die gegebene Formel unterscheidet sich von der des Peucedanins, welche $\mathrm{Hlasiwetz}$ und Weidel aufgestellt haben, durch einen Mindergehalt von $\mathrm{CH}_{2}$. Die directe Methoxylbestimmung meiner Substanz ergab den Gehalt einer $\mathrm{OCH}_{3}$ Gruppe; Hlasiwetz und Weidel nehmen an, dass das Peucedanin zwei durch Salzsäure abspaltbare Methylgruppen (Methoxyle) enthalte. Die Formel $\mathrm{C}_{14} \mathrm{H}_{10}\left(\mathrm{OCH}_{3}\right)_{2} \mathrm{O}_{2}$ würde in 100 Theilen für $\mathrm{OCH}_{3} \ldots 22 \cdot 79$ verlangen, während die $\mathrm{Be}$ stimmung $12 \cdot 12 \%$ ergab.

\footnotetext{
1 Journal für pract. Chemie, 46, 371.

2 Journal für pract. Chemie, 62, 275.

3 Ann. der Chemie, 174, 79.
} 
Eine weitere Differenz besteht im Schmelzpunkt. Die von mir erhaltene Substanz schmilzt bei $104-105^{\circ} \mathrm{C}$. (uncorr), während das Präparat, welches Hlasiwetz und Weidel untersucht haben, bei $81-82^{\circ}$ schmolz. Ich will daher die bei $105^{\circ}$ schmelzende Verbindung, deren Identität mit Peucedanin nicht ohneweiters behauptet werden kann, als Oroselonmonomethyläther weiterhin bezeichnen.

\section{Einwirkung von Salzsäure.}

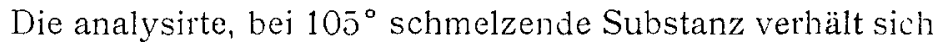
gegen Salzsäure in alkoholischer Lösung ähnlich dem von Hlasiwetz und Weidel untersuchten Peucedanin und liefert in quantitativer Ausbeute Oroselon. Behufs Darstellung desselben habe ich je $20 \mathrm{~g}$ meines Präparates in der gerade erforderlichen Menge siedenden Alkohols gelöst. In diese heisse Lösung wurde ein gleiches Volum concentrirter, reiner Salzsäure rasch eingetragen. Schon während des Eingiessens findet die Abscheidung feiner, weisser Krystallnadeln statt, die sich so rasch vermehrt, dass schliesslich das Ganze zu einem dicken Brei erstarrt. Nach dem Abkühlen wurde die Ausscheidung auf der Pumpe filtrirt und mit Wasser gevvaschen, um die anhaftende Salzsäure zu entfernen. Nach dem Abpressen stellt das Rohproduct eine blendend weisse Masse dar, die in siedendem Alkohol schwer-, fast unlöslich in kaltem Essigäther, Äther und Ligroin ist. Zur weiteren Reinigung habe ich die fein zerriebene, gut getrocknete Substanz in Benzol gelöst. Die gelblich gefärbte Lösung wurde nach dem Abkühlen mit Petroleumäther so lange versetzt, bis eine Trübung eintrat. Beim Stehen scheiden sich nun vollkommen farblose, schwach seidenglänzende, lange, verfilzte Krystallnadeln ab, die, sobald eine Vermehrung derselben nicht mehr beobachtet wurde, abgesaugt und getrocknet wurden. Der Schmelzpunkt dieses mit Oroselon unbedingt identischen Productes wurde zu $176^{\circ} \mathrm{C}$. (uncorr.) gefunden; Hlasiwet $z$ und Weidel geben den Schmelzpunkt desselben zu $177^{\circ}$ an. Die Verbrennung ergab Zahlen, welche die Identität mit Oroselon ausser Zweifel stellen.

J. $0.2064 \mathrm{~g}$ Substanz gaben $0.5184 \mathrm{~g}$ Kohlensäure und $0.0883 g$ Wasser. 
II. $0.2086 \mathrm{~g}$ Substanz gaben $0.5232 \mathrm{~g}$ Kohlensäure und $0.0893 \mathrm{~g}$ Wasser.

In 100 Theilen:

\begin{tabular}{|c|c|c|c|}
\hline \multirow{2}{*}{\multicolumn{2}{|c|}{$\begin{array}{l}\text { Berechnet für } \\
\mathrm{C}_{14} \mathrm{H}_{12} \mathrm{O}_{4}\end{array}$}} & \multicolumn{2}{|c|}{ Gefunden } \\
\hline & & $\bar{I}$ & II \\
\hline$r$ & $68 \cdot 85$ & $68 \cdot 50$ & $68 \cdot 40$ \\
\hline $\mathrm{H}$ & $4 \cdot 91$ & $4 \cdot 75$ & $4 \cdot 76$ \\
\hline
\end{tabular}

Es schien nun vor Allem wichtig, nachzuweisen, dass das Oroselon keine abspaltbare Methylgruppe enthält, und weiters war es nothwendig, eine Moleculargewichtsbestimmung dieser Verbindung auszuführen, um dadurch die früher gegebene Formel $\mathrm{C}_{15} \mathrm{H}_{14} \mathrm{O}_{4}$ zu stützen.

Was zunächst die Methoxylbestimmung angeht, so verlief dieselbe, wie zu erwarten war, negativ. Eine grössere Quantität des Oroselons mit Jodwasserstoffsäure in den Zeisel'schen Apparat eingebracht, gab selbst bei langem Erhitzen nicht die kleinste Menge Jodmethyls, so dass die vorgelegte Silberlösung vollkommen klar blieb.

\section{Moleculargewichtsbestimmung.}

Da die Bestimmungen des Moleculargewichtes mit Hilfe des Depressimeters keine übereinstimmenden Resultate ergaben, so habe ich dieselben nach der Methode von Beckmann vorgenommen. Als Lösungsmittel musste reiner Essigäther verwendet werden, weil andere Flüssigkeiten, wie Äther oder Alkohol, nicht einmal 1\% Oroselon aufzulösen vermochten.

Die Beobachtungsresultate sind in der folgenden Tabelle zusammengestellt.

\begin{tabular}{|c|c|c|c|c|c|c|c|}
\hline & $\begin{array}{c}\text { Essig- } \\
\text { ather } \\
g\end{array}$ & $\begin{array}{c}\text { Sub- } \\
\text { stanz } \\
g\end{array}$ & $\begin{array}{c}\text { Procent- } \\
\text { gehalt d. } \\
\text { Lösung }\end{array}$ & $\begin{array}{c}\text { Beob. } \\
\text { Erhöhg. } \\
\text { des Sp. }\end{array}$ & $K$ & \multicolumn{2}{|c|}{ Moleculargewicht } \\
\hline gefunden & berechnet \\
\hline I. & $23 \cdot 2750$ & 0.3782 & $1 \cdot 624$ & $0 \cdot 18^{\circ}$ & $26 \cdot 1$ & $235 \cdot 5$ & \} \\
II. & 23.2750 & 0.4936 & $2 \cdot 120$ & $0.22^{\circ}$ & $26 \cdot 1$ & $251 \cdot 5$ & 244 \\
\hline
\end{tabular}

Chemie-Heft Nr. 5. 
Der Barometerstand blieb während der ganzen Dauer der Versuche unverändert.

Diese Moleculargewichtsbestimmungen, welche im Mittel 243.5 ergaben, bestätigen die Formel des Oroselons $\mathrm{C}_{14} \mathrm{H}_{12} \mathrm{O}_{4}$, welcher das Moleculargewicht 244 zukommt.

Die Identität mit Oroselon geht auch aus dem Umstande hervor, dass mein Präparat gleich jenem, welches Hlasiwetz. und Weidel in Händen gehabt haben, eine Monoacetylverbindung liefert. Da ich darauf ausging, ein höher acetylirtes Product herzustellen, so habe ich die Einwirkung mit Essigsäureanhydrid vorgenommen, welches ich in einem bedeutenden Überschusse zur Anwendung brachte. Nach dem Abdestilliren des Anhydrids im Vacuum hinterblieb eine weisse Substanz, die in Essigäther ziemlich leicht löslich war. Das Acetylproduct krystallisirt in feinen, prächtig seidenglänzenden Nadeln aus, wenn der Lösung desselben in Essigäther so lange Petroleumäther zugesetzt wird, bis eine Trübung eintritt. Durch Wiederholung dieses Verfahrens kann die Verbindung in tadellos reinem Zustande erhalten werden. Der Schmelzpunkt wurde zu $118^{\circ} \mathrm{C}$. (uncorr.) gefunden; Hlasiwetz und Weidel geben den Schmelzpunkt ihrer Acetylverbindung zu $123^{\circ}$ an. Die beiden Körper sind trotz dieser Schmelzpunktsdifferenz, wie die Analysen zeigen, als identisch zu betrachten.

$0 \cdot 2315 g$ Substanz gaben $0 \cdot 5684 g$ Kohlensäure und $0 \cdot 1046 g$ Wasser.

In 100 Theilen:

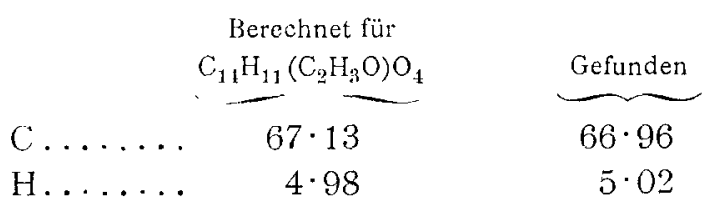

Dass hier eine Monoacetylverbindung vorliegt, beweist die directe Acetylbestimmung, die ich nach der Methode von Wenzel ${ }^{1}$ ausgeführt habe.

1 Monatshefte für Chemie, XVIII, 658. 
$0.3278 \mathrm{~g}$ Substanz gaben nach der Verseifung Essigsäure, welche $11.8 \mathrm{~cm}^{3}$ Kalilauge vom Titer 0.00556 zur Neutralisation brauchte. Daraus rechnet sich:

In 100 Theilen:

$$
\begin{aligned}
& \text { Berechnet für }
\end{aligned}
$$

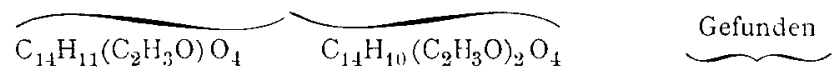

$$
\begin{aligned}
& \begin{array}{llll}
\mathrm{C}_{2} \mathrm{H}_{3} \mathrm{O} \ldots & 15 \cdot 03 & 26 \cdot 21 & 15 \cdot 36
\end{array}
\end{aligned}
$$

Versuche, die ich ausgeführt habe, um ein höher acetylirtes Derivat des Oroselons darzustellen, ergaben keine Resultate: Weder durch Erhitzen von Oroselon mit Essigsäureanhydrid bei Gegenwart von entwässertem Natriumacetat, noch durch Erhitzen unter Druck konnte das angestrebte Ziel erreicht werden, und es scheint demnach, dass das Oroselon nur eine acetylirbare Hydroxylgruppe enthält.

\section{Untersuchung der Partie $A$.}

Durch systematisches Umkrystallisiren konnte auch aus der früher mit $A$ bezeichneten, bei $84-93^{\circ}$ schmelzenden Partie eine kleine Quantität des eben besprochenen Oroselonmonomethyläthers abgeschieden werden. Die Hauptmenge aber konnte, trotzdem ich die einzelnen Fractionen, die den gleichen Schmelzpunkt besassen, vereinte und neuerdings aus Benzol, dann aus Äther umkrystallisirte, nicht in scharf schmelzende Substanzen zerlegt werden. Es verhielt sich demnach die Partie $A$ analog der mit $B$ bezeichneten, die auch nur eine verhältnissmässig kleine Quantität von reinem Oroselonmonomethyläther liefert, während die Hauptmasse den Schmelzpunkt von $93-100^{\circ}$ behielt.

Aus Krystallfractionen, die sowohl aus $A$ als $B$ erhalten wurden und die den Schmelzpunkt $87-95^{\circ}$ hatten, erhielt ich beim langsamen Abdunsten der ätherischen Lösung im Exsiccator nicht selten grosse, wohlausgebildete, spitze, sechsseitige Prismen, die eine schwach gelblichweisse Farbe und lebhaften Glasgları zeigten. Dieses schön krystallisirte Product hat Herr Hofrath v. Lang einer krystallographischen Untersuchung 
unterzogen, für welche ich ihm zu grossem Danke verpflichtet bin; er theilt darüber Folgendes mit:

"Die Krystalle haben die Form von langen, sechsseitigen Prismen (Deuteroprisma 110), deren Längskanten bisweilen abgestumpft sind (Protoprisma 122). Die Enden werden durch die schuppigen Flächen eines flachen Rhomboëders (100) gebildet, die unter $23^{\circ} 40^{\prime}$ zu einander geneigt sind. Für das hexagonale System spricht auch das optische Verhalten, indem die Krystalle einaxig sind mit negativem Charakter".

Die Verbindung zeigt den unscharfen, zwischen $85-93^{\circ} \mathrm{C}$. liegenden Schmelzpunkt und gab, der Analyse unterworfen, folgende Zahlen:

I. $0 \cdot 3405 \mathrm{~g}$ Substanz gaben $0.8795 \mathrm{~g}$ Kohlensäure und $0 \cdot 1760 \mathrm{~g}$ Wasser.

II. $0.2542 g$ Substanz gaben $0.6588 g$ Kohlensäure und $0 \cdot 1330 \mathrm{~g}$ Wasser.

In 100 Theilen:

$$
\begin{array}{crr} 
& \text { I. } & \multicolumn{1}{c}{\text { II. }} \\
\text { C . . . } & \multicolumn{1}{c}{{ }^{0} \cdot 42} & 70 \cdot 68 \\
\text { H. . . . } & 5 \cdot 72 & 5 \cdot 88
\end{array}
$$

Diese Werthe stimmen überein mit den für die Formel $\mathrm{C}_{16} \mathrm{H}_{16} \mathrm{O}_{4}$ gerechneten und jenen, welche Bothe, $\mathrm{Heut}$, $\mathrm{H}$ lasiwet $z$ und Weidel gefunden haben.

In 100 Theilen:

$$
\begin{aligned}
& \text { Berechnet für Gefunden } \\
& \mathrm{C}_{16} \mathrm{H}_{16} \mathrm{O}_{4} \quad \text { im Mittel } \\
& \begin{array}{lll}
\text { C } \ldots \ldots .70 & 70.58 & 70.55
\end{array} \\
& \text { H..... } 5 \cdot 88 \quad 5 \cdot 80
\end{aligned}
$$

Die Methoxylbestimmung jedoch ergab ein Resultat, welches mit dieser Formel nicht vereinbarlich erscheint. $0 \cdot 2924 \mathrm{~g}$ Substanz gaben $0 \cdot 2867 \mathrm{~g}$ Jodsilber.

In 100 Theilen: 
Dieser Werth ist wesentlich höher als der von der Formel des Oroselonmonomethyläthers verlangte, und viel zu nieder für einen Dimethyläther des Oroselons (Peucedanin), wie die folgende Zusammenstellung zeigt:

In 100 Theilen:

$$
\overbrace{\mathrm{CCH}_{14} \mathrm{H}_{11}\left(\mathrm{OCH}_{3} \mathrm{O}_{3}\right.}^{\text {Berechnet für }} \underbrace{22 \cdot 78}_{\mathrm{C}_{14} \cdot \mathrm{C}_{10}\left(\mathrm{OCH}_{3}\right)_{2} \mathrm{O}_{2}} \underbrace{\text { Gefunden }}_{13 \cdot 24}
$$

Da aber auch diese Substanz bei Behandlung mit Salzsäure Oroselon liefert, so muss man wohl annehmen, dass dieses Product trotz seines schön krystallisirten Zustandes ein Gemisch von Oroselonmonomethyläther mit einer anderen Substanz, vielleicht Oroselondimethyläther, darstellt, welches durch fractionirte Krystallisation nicht $z$ u trennen ist.

Ebenso unscharfe Resultate ergab bei der Analyse die bei $77-81^{\circ} \mathrm{C}$. schmelzende, durch fractionirte Krystallisation erhaltene Partie. Stets waren die Verbrennungszahlen den für Peucedanin gerechneten naheliegend, waren aber für den Oroselonmonomethyläther viel zu niedrig.

Eine spätere Untersuchung müsste hier anknüpfen und man müsste durch Auffindung neuer Trennungsmethoden die Abscheidung des Oroselondimethyläthers, welcher wahrscheinlich in diesen unscharf schmelzenden Producten enthalten ist, herbeiführen.

An dieser Stelle sei es mir gestattet, meinem verehrten Lehrer, Herrn Prof. Weidel, meinen innigsten Dank für die werkthätige Unterstützung auszusprechen, welche er mir während der Ausführung dieser Arbeit angedeihen liess. Auch Herrn Hofrath v. Lang, sowie Herrn Dr. Hockauf drücke ich meinen Dank aus für die Bereitwilligkeit, mit welcher die genannten Herren die krystallographischen, beziehungsweise botanischen Untersuchungen ausgeführt haben. 